\title{
Materials Characterization by Raman Microscopy
}

\author{
Steva Lević
}

\begin{abstract}
By using Raman microscopy it is possible to obtain various information such as chemical composition of material, its morphological and mechanical properties, distribution of specific compounds, structure of materials, etc. In order to localize the presence of specific compound in the complex mixtures such as in the case of solid capsules containing active compounds it is necessary to process its spectra.
\end{abstract}

In recent years Raman microscopy has attracted great attention as suitable technique for characterization of different solids and composites. By using Raman microscopy it is possible to obtain various information such as chemical composition of sample, its morphological properties, distribution of specific compounds, structure of materials, mechanical properties of sample, etc. The main advantages of Raman microscopy are reliability, generally easy data interpretation, non-destructive analysis of sample, possibilities for mapping of sample surface as well as deep profile analysis (Hollricher 2011, Fries and Steele 2011).

\section{How to cite this book chapter:}

Lević, S. 2019. Materials Characterization by Raman Microscopy. In: Vucelić Radović, B., Lazić, D. and Nikšić, M. (eds.) Application of Molecular Methods and Raman Microscopy/Spectroscopy in Agricultural Sciences and Food Technology, Pp. 187-192. London: Ubiquity Press. DOI: https://doi.org/10.5334/bbj.o. License: CC-BY 4.0 
The analysis of pure compounds is recommended before performing any chemical process or compounds mixing. Solid samples like crystals or powders can be analysed by simple deposition of material on the microscopic slide. Results could be obtained in the maters of seconds (Fig.1).

The Raman spectrum of ethyl vanillin (Fig. 1) exhibits several strong bands that are in agreement with chemical structure of this flavour compound. Usually, for material characterization the most important part of the spectrum is "fingerprint region", i.e. the part of spectrum with characteristic bands. For ethyl vanillin, "fingerprint region" could be defined in the spectral range $1000-1800 \mathrm{~cm}^{-1}$, where main bands are mainly associated with vibrations of the benzene ring.

Materials with crystal structure (like ethyl vanillin) can be relatively easily analyzed by Raman spectroscopy since the spectrum can be collected under short acquisition time and at maximum laser power.

When the spectra of pure compounds are known then it is possible to localize the presence of compounds in the complex mixtures. This is especially suitable in the case of formation of solid capsules that contain active compounds (e.g. encapsulated active compound). The efficiency of encapsulation process could be analysed using Raman microscopy in combination with mapping of particles' surface (Fig. 2).

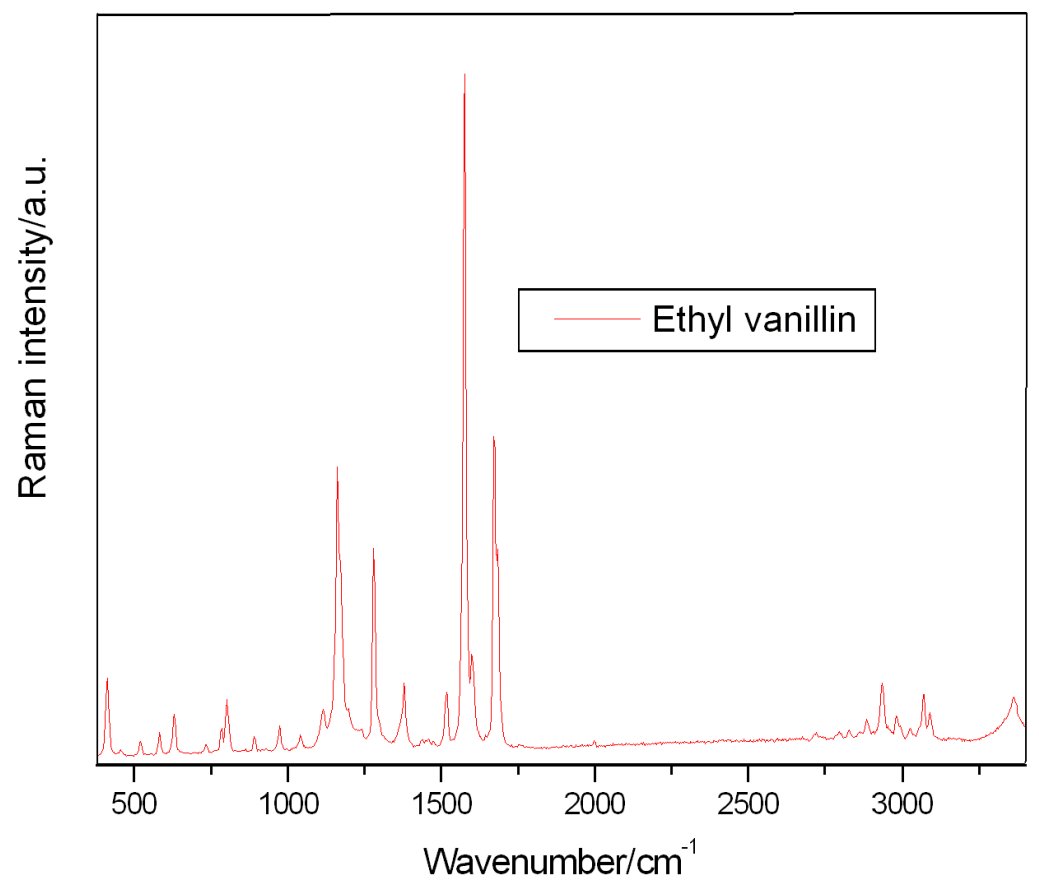

Figure 1: Raman spectrum of ethyl vanillin. Acquisition time 1s, laser $532 \mathrm{~nm}$. 

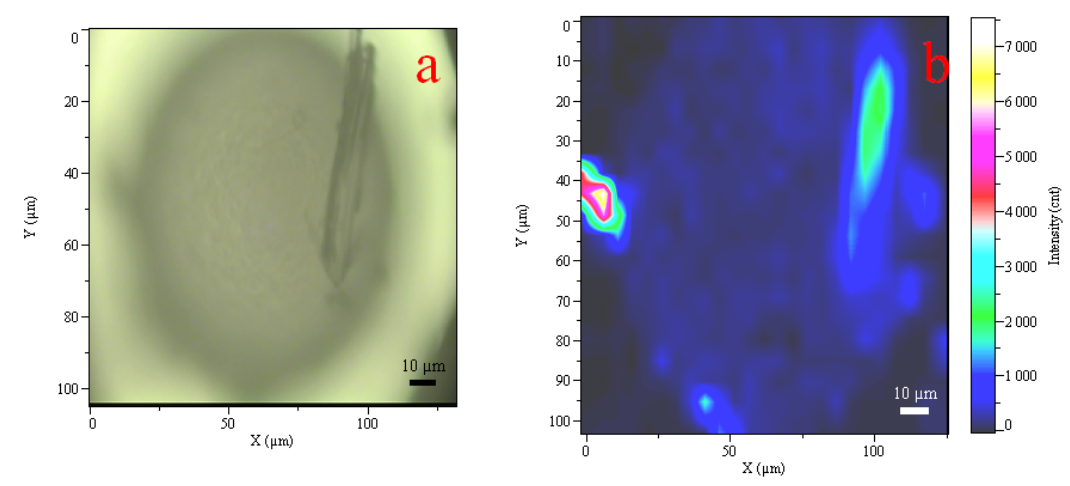

Figure 2: Raman mapping of carnauba wax microparticles and free ethyl vanillin that is not encapsulated into structure of carrier material (i.e. wax). Acquisition time 1s, step $1 \mu \mathrm{m}$, laser $532 \mathrm{~nm}$.

The free flavour is visible in the form of crystals on the particle surface. However, spherical shape of the particle prevents simultaneous identification of all crystals on the particle surface by light microscopy (Fig. 2a). Using Raman mapping it is possible to overcome this problem and as a result more visible ethyl vanillin crystals could be observed (Fig. 2b). The mapping spectra were collected using acquisition time of $1 \mathrm{~s}$ and data were showed as maximum at $1576 \mathrm{~cm}^{-1}$ (the most intensive band in the Raman spectrum of ethyl vanillin).

However, some analyses require longer acquisition time in order to ensure useful Raman spectra. In the Fig. 3 the two spectra of the same sample collected at two different acquisition times are showed. As can be seen, the longer acquisition time enables better visibility of bands at around $2900 \mathrm{~cm}^{-1}(\mathrm{CH}$ vibrations) which are important for cellulose characterisation (Agarwal et al. 2010).

Longer acquisition time is required when the intensity of main bands are not so strong and good for interpretation. In this case, Raman microscopy was found to be excellent for characterization of composite materials and successful determination of differences that occurred during sample preparation.

Characterization of natural polymers could be efficiently carried out by using Raman microscopy. As can be seen in the Fig. 4 Raman spectrum of sodium alginate exhibits several intensive bands that are linked to its structure and molecular properties. The use of Raman microscopy for such analyses usually requires optimisation of measuring method in order to provide useful data that can be further interpreted (see below).

Several key parameters must be adjusted before final sample analysis. The main parameter is selection of laser wavelength that will be used for analysis. Many materials exhibit strong fluorescence, especially when laser such as $532 \mathrm{~nm}$ laser is used. Generally, in the case of high fluorescence it is recommended to use laser of higher wavelength (e.g. $785 \mathrm{~nm}$ or $1064 \mathrm{~nm}$ ). 


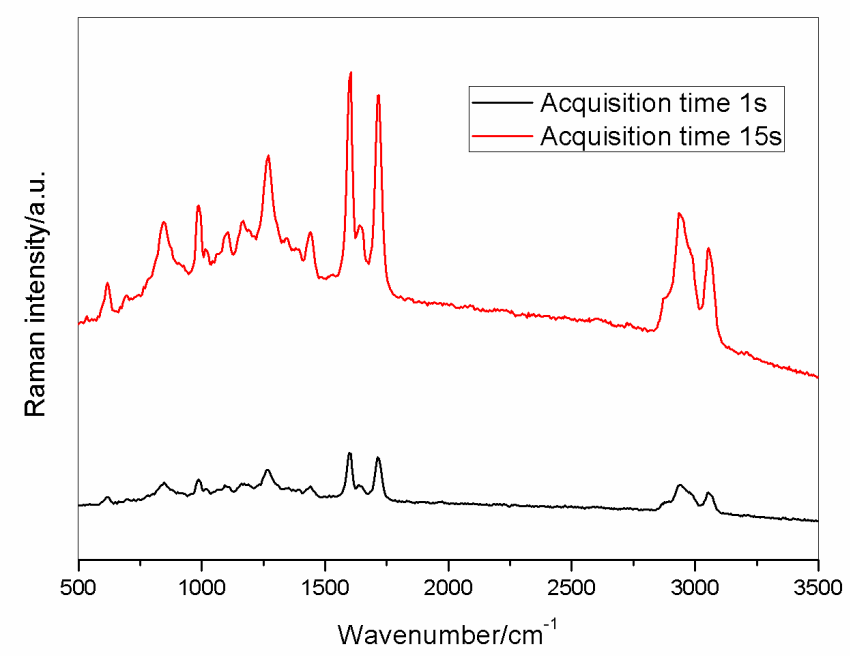

Figure 3: Influence of acquisition time on the spectra of cellulose based composite material. Laser $532 \mathrm{~nm}$.
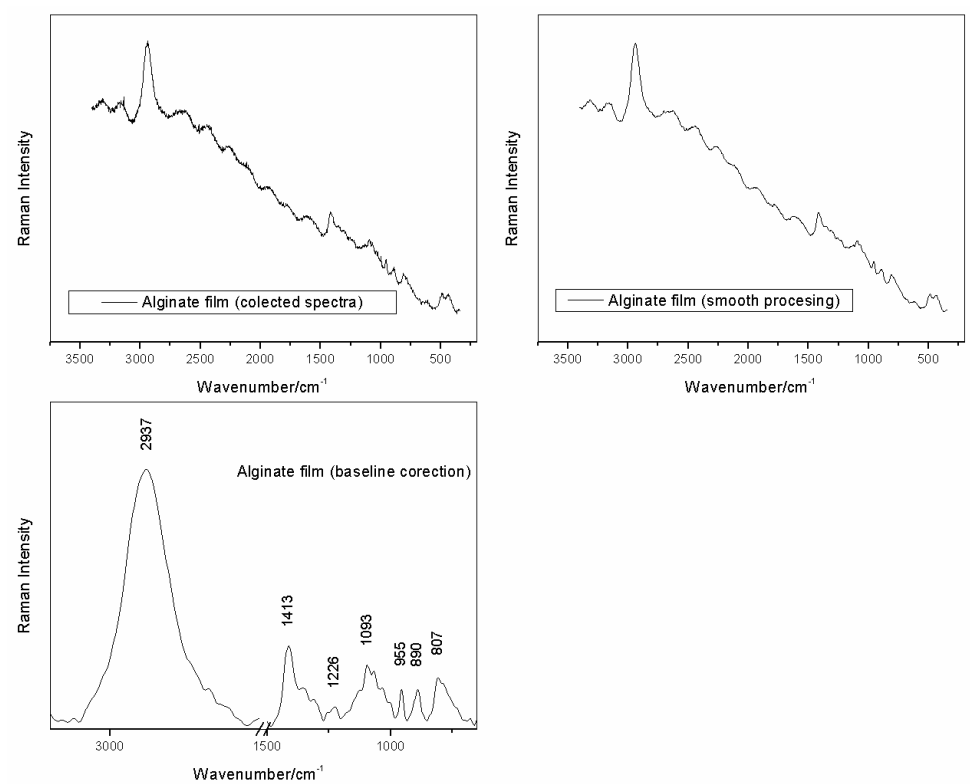

Figure 4: The post-analysis treatments of sodium alginate Raman spectra. Acquisition time 1s, laser $532 \mathrm{~nm}$. 
The data collected during Raman microscopy usually require some sort of treatment and interpretation before final conclusions. In the Fig. 4 the basic and processed Raman spectra of sodium alginate film are showed.

In the case of sodium alginate, the important bands are at $1413 \mathrm{~cm}^{-1}$ (carboxylate group), while the bands between $1300-800 \mathrm{~cm}^{-1}$ are related to the internal structure of alginate molecule and specific ratios of alginate subunits (CamposVallette et al. 2010).

The obtained spectrum of pure sodium alginate is subsequently processed by smoothing interpolation and baseline correction in order to eliminate as much as possible of data that do not belong to spectrum of alginate. Finally, processed spectrum obtained after baseline corrections and peaks identification provides data that could be used for further analyses.

The processing of obtained (basic) spectra can be divided in following operations:

1. Smoothing;

2. Baseline correction;

3. Intensity normalization;

4. Removing of suspicious points (i.e. peaks) form spectra.

These treatments are usually called a "pre-processing" of data and are required steps, especially when further analyses are necessary (e.g. statistical data analyses).

When an unknown compound is analysed, then the spectra of similar compounds could be found online (i.e. in the online bases of spectral data) and compared with analysed sample (http://sdbs.riodb.aist.go.jp/sdbs/cgi-bin/ direct_frame_top.cgi). This could be useful for identification of compounds and results interpretation.

\section{References}

Agarwal, U.P., Richard S. Reiner, R.S., \& Ralph, S.A. (2010). Cellulose I Crystallinity Determination Using FT-Raman Spectroscopy: Univariate and Multivariate Methods. Cellulose, 17, 721-733.

Campos-Vallette, M.M., Chandía, N.P., Ernesto Clavijo, E., Leal, D., Matsuhiro, B., Osorio-Román, I.O., \& Torres, S. (2010). Characterization of Sodium Alginate and Its Block Fractions by Surface-Enhanced Raman Spectroscopy. Journal of Raman Spectroscopy, 41, 758-763.

Fries, M., \& Steele, A. (2011). Raman Spectroscopy and Confocal Raman Imaging in Mineralogy and Petrography. In T. Dieing, O. Hollricher, \& J. Toporski, J. (Eds). Confocal Raman Microscopy (pp. 111-135). Springer-Verlag, Berlin, Heidelberg. DOI: https://doi.org/10.1007/978-3-642-12522-5_6 
Hollricher, O. (2011). Raman Instrumentation for Confocal Raman Microscopy. In T. Dieing, O. Hollricher, J. Toporski, J. (Eds), Confocal Raman Microscopy (pp. 43-60). Springer-Verlag, Berlin, Heidelberg. DOI: https:// doi.org/10.1007/978-3-642-12522-5_3.

Spectral Database for Organic Compounds, AIST (SDBS). Retrieved from http://sdbs.riodb.aist.go.jp/sdbs/cgi-bin/direct_frame_top.cgi 